\title{
Organization of membrane motor in outer hair cells: an atomic force microscopic study
}

\author{
Ghanshyam P. Sinha • Firouzeh Sabri • \\ Emilios K. Dimitriadis • Kuni H. Iwasa
}

Received: 18 August 2009 /Revised: 17 September 2009 / Accepted: 22 September 2009/Published online: 7 October 2009

(C) The Author(s) 2009. This article is published with open access at Springerlink.com

\begin{abstract}
Using atomic force microscopy, we imaged the cytosolic surface of the lateral plasma membrane of outer hair cells from guinea pigs' inner ear. We used a "cell-free" preparation, in which a patch of plasma membrane was firmly attached to a substrate and the cytoplasmic face was exposed. The membrane patches contained densely packed particles whose diameter, after correcting for the geometry of the probing tip, was $\sim 10 \mathrm{~nm}$. The particles were predominantly aligned unidirectionally with spacing of $\sim 36 \mathrm{~nm}$. The density of the particle was $\sim 850 \mu \mathrm{m}^{-2}$, which could be an underestimate presumably due to the method of sample preparation. Antibody-labeled specimens showed particles more elevated than unlabeled preparation indicative of primary and secondary antibody complexes. The corrected diameters of these particles labeled with anti-actin were $\sim 12 \mathrm{~nm}$ while that with antiprestin were $\sim 8 \mathrm{~nm}$. The alignment pattern in antiprestin-labeled specimens resembled that of the unlabeled preparation. Specimens labeled with actin antibodies did not show such alignment. We interpret that the particles observed in the unlabeled membranes correspond to the $10-\mathrm{nm}$ particles reported by electron microscopy and that these particles contain prestin, a
\end{abstract}

\footnotetext{
G. P. Sinha $\cdot$ F. Sabri $\cdot$ K. H. Iwasa $(\bowtie)$

Biophysics Section, Laboratory of Cellular Biology,

National Institute on Deafness and Other Communication Disorders,

National Institutes of Health,

5 Research Court,

Rockville, MD 20850-3211, USA

e-mail: iwasa@nih.gov

E. K. Dimitriadis

Laboratory of Bioengineering and Physical Sciences,

National Institute of Biomedical Imaging Bioengineering,

National Institutes of Health,

13 South Drive,

Bethesda, MD 20892-5766, USA
}

member of the SLC26 family, which is essential for electromotility.

Keywords Outer hair cells · Atomic force microscopy . Prestin · Cytosolic plasma membrane · Hair Cell . Microscopy $\cdot$ Mechanoelectrical transduction . Immunocytochemistry

\section{Introduction}

Electromotility $[2,7,28]$ is a conspicuous motile property of cochlear outer hair cells (OHCs) and is driven by electrical energy available at the plasma membrane. Along with hair bundle motility $[8,30]$, this motility of the cell body is capable of producing fast mechanical force in response to mechanical stimuli and thereby amplifying the mechanical signal. It is therefore a critical factor for the sensitivity, frequency selectivity, and nonlinear characteristics of the mammalian ear $[9,35]$.

Electrophysiological studies showed that motile activity is associated with charge transfer across the membrane [3, 43], similar to piezoelectricity $[14,19,27,38]$. The mobile charge contributes to the membrane capacitance as a nonlinear, voltage-dependent component. According to two-state models, the steepness of the voltage dependence indicates a unit mobile charge of about $0.9 \mathrm{e}$ and the ratio of the total charge transfer to the unit charge gives a motor unit density between 5,000 and $10,000 \mu^{-2}$ in OHCs from the middle turns of the cochlea $[29,43,45]$. The transfer of this unit mobile charge is coupled with area change of about $4 \mathrm{~nm}^{2}$ [1].

A molecular biological approach identified prestin, a member of the SLC26 family of anion transporters, as the protein associated with the motile activity of the lateral wall. Cells transfected with prestin show nonlinear capacitance, 
characteristic of OHCs. The steepness of the voltage dependence is about the same as OHCs [41, 57], and the corresponding area change is about $2 \mathrm{~nm}^{2}$ [12]. As is often the case with membrane proteins, prestin appears to form oligomers $[40,58]$, presumably about $10 \mathrm{~nm}$ in diameter [37, 39].

Morphological studies with electron microscopy showed that the lateral plasma membrane is packed with particles of about $10 \mathrm{~nm}$ in diameter (the "10-nm particles") [17, 21, 29, 45]. These particles are not apparent from the extracellular face with freeze fracture method without etching [29]. The reported density of those particles ranges between 2,500 and $6,000 \mu \mathrm{m}^{-2}[17,29,45]$. Although the density of the 10 -nm particles is similar to that of the motor molecule determined from electrophysiological experiments in the order of magnitude, the relationship between the 10-nm particle and the functional unit remains unclear because their ratio shows significant uncertainty and variability [45].

An important question that has not been directly addressed, even though assumed in some of the studies $[37,39]$, is the molecular identity of the $10-\mathrm{nm}$ particles, specifically whether or not prestin is a significant constituent of the 10-nm particles. Prestin forms oligomers, which are consistent with the dimension of the 10-nm particles, in the plasma membrane of prestin-transfected cells. However, there is no direct proof that prestin oligomers are indeed 10-nm particles, because the lateral plasma membrane of OHCs and the plasma membranes of cultured cells should contain membrane proteins other than prestin. In addition, different cytoskeletal undercoatings of these membranes could affect the organization of the membrane proteins. Specifically, the undercoating of the lateral plasma membrane of OHCs is elaborate and unique [24]. For those reasons, the 10-nm particles in OHCs and oligomers in the plasma membrane of prestin transfected cells may not be identical. In addition, the issue of oligomerization of prestin itself may not be very simple. Although studies using gel electrophoresis show the involvement of disulfide bonds in dimerization [52, 58], suggesting relative stability of oligomers, studies that use fluorescence resonance energy transfer (FRET) show that dimerization of prestin is sensitive to cholesterol content in the plasma membrane and that the FRET signal increases or decreases with cholesterol content of intact OHCs [42]. This observation suggests that prestin monomers may exist in the plasma membrane of OHCs. Those reports therefore pose an additional question as to whether prestin exists outside of the 10-nm particles even if the $10-\mathrm{nm}$ particles contain prestin.

In this study, we specifically probe the molecular identity of these 10-nm particles by using atomic force microscopy (AFM). To obtain the spatial resolution required, the extracellular side of the plasma membrane must adhere firmly to a flat substrate and the cytosolic side must be exposed. Although an earlier report shows an AFM image of such a patch of the plasma membrane that is broken off from a fixed outer hair cell [33], the image is too complex to analyze, apparently being dominated by intracellular structure such as the cortical cytoskeleton. Thus, it seems necessary to tear off a patch of membrane before fixing the cell. Such a method, called "cell-free" preparation, has been successfully used for tissue-cultured cells [4, 23, 39]. Here, we apply this method on acutely dissociated outer hair cells and image the membrane patch with AFM.

We also tested the immunoreactivity with prestin antibodies by examining the sizes and organization of the molecular complexes that these antibodies form. We show that imaging the bound antibodies at the single molecule scale with the atomic force microscope, a relatively unexplored method, has the advantage of viewing the arrangement of the antigen-antibody complexes when compared with the current optical methods in use for immunohistochemistry.

\section{Materials and methods}

\section{Cell-free preparation}

Outer hair cells were dissociated from the organ of Corti of pigmented guinea pigs (Elm Hill Labs, Chelmsford, MA, USA) between 200 and $400 \mathrm{~g}$ in body mass following a method previously described [13] and approved as protocol 1195-05 NINDS/NIDCD. Briefly, the organ of Corti was dissected from the cochlea in a perilymph-like medium, which contained $140 \mathrm{mM} \mathrm{NaCl}, 5 \mathrm{mM} \mathrm{KCl}, 2 \mathrm{mM} \mathrm{MgCl} 2$, $1.5 \mathrm{mM} \mathrm{CaCl}$, $10 \mathrm{mM}$ 4-(2-hydroxyethyl)-1-piperazinee thanesulfonic acid, $2 \mathrm{mM}$ Na pyruvate, $2 \mathrm{mM}$ creatine, and $2 \mathrm{mM} \mathrm{Na}$ ascorbate with $\mathrm{pH}$ adjusted to 7.3 with $\mathrm{NaOH}$, which we refer to as the standard medium. The Hensen cells were then removed with a needle. The tissue obtained was then treated with $1 \mathrm{mg} / \mathrm{ml}$ dispase (Worthington) for $15 \mathrm{~min}$ at $23^{\circ} \mathrm{C}$ before being gently triturated three times with a plastic pipette. The isolated cells were then placed in a chamber created on an aldehyde-coated glass slide (superaldehyde substrates, ArrayIt Microarray Technology, Sunnyvale, CA, USA), to which a thin plastic film with a printed grid, with $1 \mathrm{~mm}$ spacings, was glued to the slide underside. The chamber was centrifuged at $200 \times g$ for $20 \mathrm{~min}$. The positions of isolated cells with good optical density were recorded with reference to the grid so that these locations could be examined later for isolated membrane patches (cell-free preparation). This preparation was then briefly (for about $0.1 \mathrm{~s}$ ) sonicated using an ultrasonic cell disrupter (XL-2000, Misonix, Farmingdale, NY, USA) at a low power setting to shear open the cells. The chamber was then carefully rinsed with the standard medium to remove floating cellular debris. 


\section{Unlabeled specimens}

To obtain images of unlabeled specimens, the prepared samples as described above were immediately treated with $2.5 \%$ glutaraldehyde for $10 \mathrm{~min}$. They were then washed with the standard medium and placed on the AFM Bioscope stage for scanning.

\section{Antiprestin-labeled specimens}

To examine the immunoreactivity of the preparation, we used prestin antibodies (provided by Dr. B. Kachar, NIDCD, NIH) that were raised in rabbits against a peptide from the $\mathrm{C}$ terminus domain of gerbil prestin (amino acids 725-744) [5]. The sonicated and rinsed samples were treated with antiprestin primary antibodies at $\sim 5 \mu \mathrm{g} / \mathrm{ml}$ concentration for $30 \mathrm{~min}$ [5]. Then, the samples were washed three times before being treated with fluoresceinconjugated secondary antibodies (Amersham Pharmacia Biotech, Arlington Heights, IL, USA) at a 1:200 dilution for $20 \mathrm{~min}$. These samples were rinsed three times before fixation with $2.5 \%$ glutaraldehyde. This was followed by several rinses, and then the samples were scanned with AFM. Antibodies were tested for their specific immunoreactivity on the organ of Corti by immunofluorescence labeling before use.

\section{Anti-actin and anti-DNase II-labeled specimens}

Actin antibodies (A5060, Sigma-Aldrich) and DNase II antibodies (D1689, Sigma-Aldrich) raised in rabbit were used as control antibodies. The protocol was the same as the one for antiprestin-labeled specimens except that $\sim 20 \mu \mathrm{g} / \mathrm{ml}$ actin antibodies or $0.25 \mu \mathrm{g} / \mathrm{ml}$ DNase II antibodies were used instead of prestin antibodies.

\section{Atomic force microscopy}

Bioscope AFM (Veeco, Santa Barbara, CA, USA) mounted on an inverted fluorescence microscope (Eclipse TE300, Nikon) was used with a Quadrexed Nanoscope IIIa controller. All the measurements were done in fluid tapping mode with V-shaped cantilevers mounted on a fluid holder. The standard medium was used as imaging buffer. In most cases, we used Si-N cantilevers, $200 \mu \mathrm{m}$ in length, with a spring constant $\approx 0.06 \mathrm{~N} / \mathrm{m}$ and oxide sharpened tips (DNP-S, Veeco, Santa Barbara, CA, USA). The feedback gains and scan speed were optimized before scanning. The typical amplitude of tip vibration was set between 0.5 to $1 \mathrm{~V}$ at scan speeds of $\sim 0.8 \mathrm{~Hz}$ per pair of scan lines. The tapping frequency was about $8 \mathrm{kHz}$, slightly lower than the cantilever resonance frequency, such that the amplitude of oscillation at the selected frequency was $5-10 \%$ below the amplitude at resonance frequency. All scans shown were performed from left to right, where the height profiles and phase contrast images were recorded simultaneously. Height profiles revealed the topography of the scanned surface, whereas phase contrast images originated from the phase lag of the oscillating tip following differences in height and variations in the probe sample interaction.

Data acquisition and analysis

Images were obtained with the Nanoscope software (Veeco, Santa Barbara, CA, USA) at maximum resolution of $512 \times$ 512 pixels. Some processing and analyses of the images were done using the same program. The original images were flattened to eliminate background slopes and variations between scan lines due to thermal drift. Low-pass filters were also applied to remove small spikes (usually $<1 \mathrm{~nm}$ ) that often appear in the images due to thermal drift of the scanner and environmental noise. To analyze patterns in an image obscured by noise, we used a digitized version of the autocorrelation function $S$. For an image $\mathrm{f}$ of $M \times N$ pixels, it is given by [48],

$S(x, y)=g(x, y) \sum_{i=1}^{M} \sum_{j=1}^{N} f(i, j) f(i+x, j+y)$,

with a normalization factor,

$$
g(x, y)=\frac{M N}{(M-x)(N-y) \sum_{i=1}^{M} \sum_{j=1}^{N} f^{2}(i, j)} .
$$

Here, $x$ and $y$ represent position translations in the two directions. Prominent peaks in $S$ indicate the directions and spacing of periodicities.

To reduce noise in the autocorrelation function of images, we preprocessed images using two methods. One is background subtraction. Images processed with instrument software were converted to gray-scale images before imported to ImageJ or Matlab. A background image of each image was then created with Gaussian blurring, and then it was subtracted from its original image using ImageJ (Wayne Rasband, NIH).

A more elaborate processing, which we call "reconstruction", was performed on noisier images after background subtraction. First, an identical circle was fit to each raised feature (particles) using granulometric filters (an ImageJ plug-in by Dimiter Prodanov). Using MATLAB, we then created a new image that consisted of identical hemispheres, each of which was placed at the center of each circle created in the previous step. The size of these hemispheres was set to the average diameter of the particles of our interest. These reconstructed images should retain 
information about particle positions. The size of the particles in the collected images is a dilated version of the actual particles due to convolution with the finite-sized imaging probe. In order to obtain the size of the particles, a good estimate of the probe radius is necessary. Mathematical morphology describes an image surface as a dilation of the actual sample surface by the inverted tip $[15,53,56]$. Blind reconstruction methods, derived by these authors, can determine an upper bound (i.e., the bluntest of all possible probes) on the tip geometry from an image of a sample without a priori knowledge of the object's actual geometry. This is achieved by calculating the upper bound (bluntest tip) consistent with each point in the image and then choosing as the final tip the tightest envelope encompassing all the upper bounds. Such an estimate of probe geometry can be obtained by an iterative function given as $[53,56]$,

$p_{j+1}(x)=\min _{\mathbf{x}^{\prime} \in D_{I}}\left(\max _{\mathrm{d} \in D_{p^{\prime}}}\left\{\min \left[i\left(x+x^{\prime}-d\right)-i\left(x^{\prime}\right)+p_{j}(d), p_{j}(x)\right]\right\}\right)$.

The vectors $\mathbf{x}$ and $\mathbf{x}^{\prime}$ represent the horizontal plane, and $p$ and $i$ are functions at these points, which represent the inverted probe surface and image surface respectively. $p_{j}$ is the surface of the probe obtained after $j$ iterations starting from initial probe surface $p_{0}$. The extremum operation is implied to extend over all in-plane coordinates of the respective domains. $D_{I}$ is the domain of the image surface $I$. $D_{P^{\prime}}$ is the domain of $P^{\prime}$ determined by considering all possible touch points, which have the apex on or below the image surface. $\mathbf{d}$ is the displacement vector between the touch points of the tip on the image and tip apex. The maximum operation within curly bracket is a union of the corresponding revised tip shapes for all possible d's. The final tip is obtained from the best fit of the probes over the whole image as given by the minima operation over the domain of $I$. In this paper, a commercially implemented blind reconstruction procedure (SPIP, Image Metrology A/S, Denmark) was used to obtain the radius of the sphere equivalent to the tip curvature of the probe (sphere fit) together with the angle of the cone, which represents the gross shape of the probe. The images on which the procedure was applied were beforehand flattened and Gaussian filtered $(5 \times 5$ kernel, cut off wavelength $5 \mathrm{~nm})$ to remove noise contributions that may interfere with good tip estimation.

\section{Results}

The preparation

To our knowledge, cell-free preparations so far reported were formed from tissue-cultured cells [4, 23, 39]. Our preparation differed from those reports in that it was made from acutely dissociated cells, which were not homogeneous. Our method of isolating outer hair cells could not completely remove Deiters' cells or pillar cells. However, these supporting cells had turned into ghosts because their plasma membranes had broken during isolation, being firmly attached to the basilar membrane. Only outer hair cells retained unbroken plasma membranes. The percentage of outer hair cells forming strong bonds to the substrate during centrifugation is thus expected to be very high. The origin of isolated membranes in the cell-free preparation was identified by the location of the cells prior to sonication (see "Materials and methods" section). Such "cell-free" candidates are difficult to identify by standard fluorescent tagging techniques due to the short path length of the light source through the thin membrane. The membrane patches were selected out of several AFM scans of the premarked positions, where a relatively smooth elevated structure could be observed. Images with fiber like structure that could be part of the cortical cytoskeleton were excluded. Eleven unlabeled patches were obtained from different experiments, and a total of five were analyzed for particle size, height, and organization.

Examples of cell-free preparation are shown in Fig. 1. The extracellular side of the plasma membrane was bound to the aldehyde-coated glass substrate. The cytosolic face of the membrane was scanned with AFM. The height of the membrane surface varied between $8.1 \pm 1.7 \mathrm{~nm}(N=45$; height of dips) and $11.7 \pm 2.2 \mathrm{~nm}(N=45$; height of peaks; see Fig. 1d) with respect to the substrate. The value of $8.1 \mathrm{~nm}$ can be interpreted as the thickness of the plasma membrane, from the aldehyde-coated substrate. This value is compatible with the thickness between 6 and $10 \mathrm{~nm}$ of typical biological membranes with high densities of membrane proteins [6]. AFM measurements of the thickness of other types of membranes have also given similar results, as for example, thickness of purple membranes of Halobacterium salinarum is $6.3 \mathrm{~nm}$ [50]. The value of $11.7 \mathrm{~nm}$ corresponds to the height of the particles from the substrate. This value is also compatible with a recent result that prestin can protrude to the extracellular side by $\sim 2 \mathrm{~nm}$ [37]. Since those authors [37] assumed $5 \mathrm{~nm}$ for the thickness of lipid bilayer, the total thickness of the membrane including the protrusion is $7 \mathrm{~nm}$, comparable to our value.

\section{Ten-nanometer particles}

The topography (Fig. 2a) and the phase images (Fig. 2b) revealed membrane-associated particles distributed throughout the cytosolic surface. Of these two images, particles were more distinct in the phase images, which reflect local variations in height and sample's surface properties, than 
Fig. 1 Examples of cell-free preparation of OHC's lateral plasma membrane: a, b topographical AFM images of unlabelled cytosolic face. c Topographical image of actin antibody-labeled specimen. Primary actin antibodies were conjugated to secondary antibodies for enhancement. All specimen were fixed with $2.5 \%$ glutaraldehyde. The color coding for relative height is shown under each image. d Height profile along the line in a. e Height profiles along the lines in $\mathbf{b}$ and $\mathbf{c}$ are represented by a dashed line and a solid line, respectively
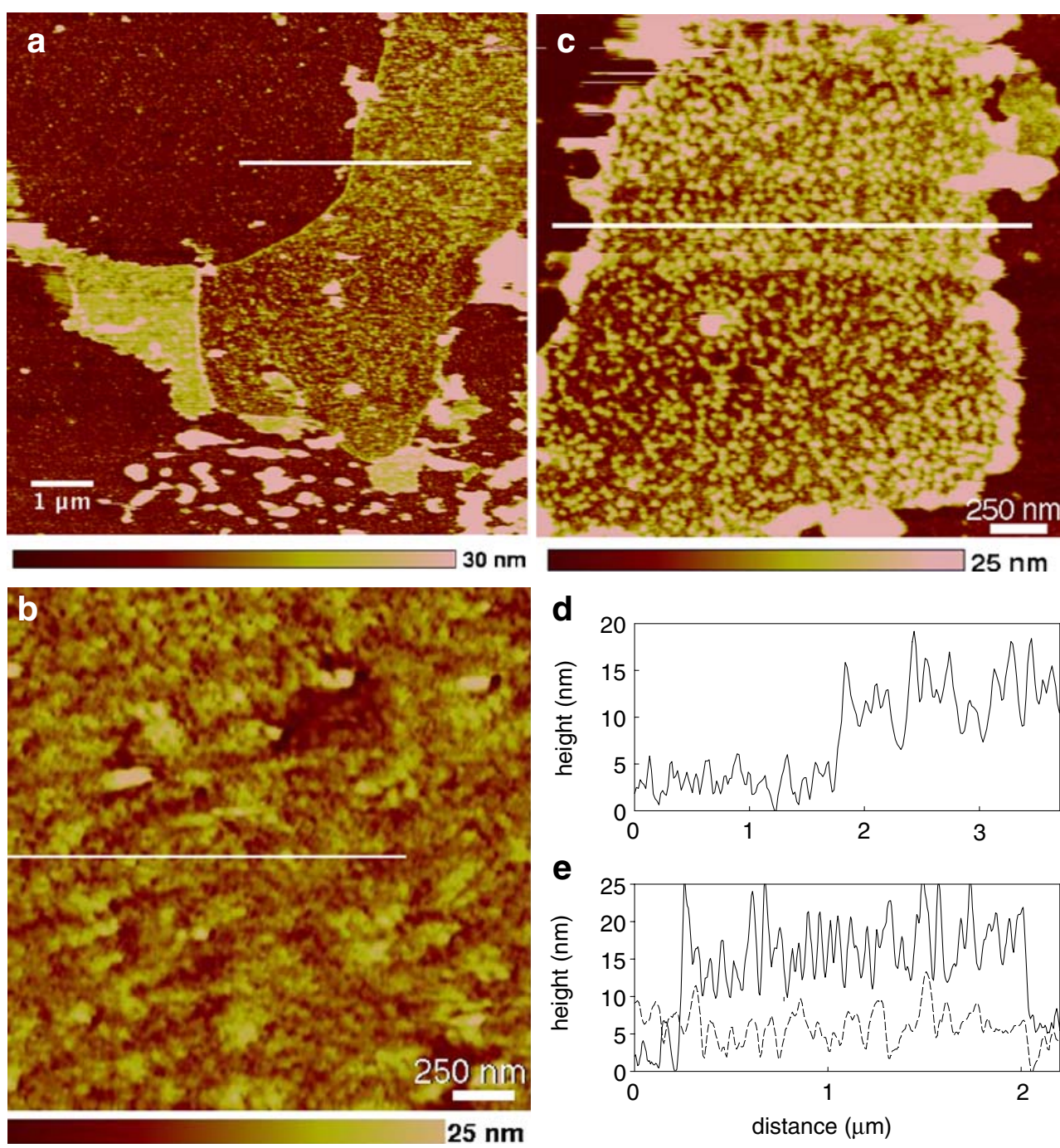
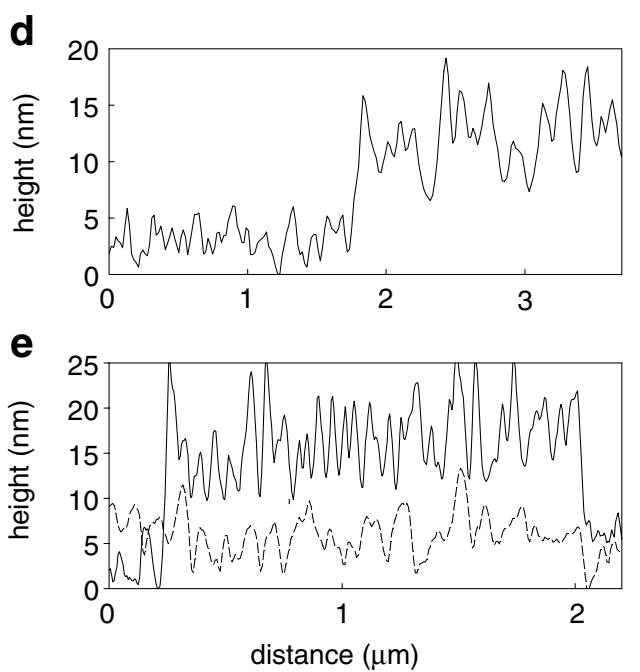

topographic images, which display local height variations. In the case of the height image, the roughness analysis (Veeco Software) showed that the maximum height range $R_{\max }$ (vertical distance between the highest and lowest data points) in the image (Fig. 2a) following plane fit and Gaussian filtering (kernel size $5 \times 5$, cutoff wavelength $5 \mathrm{~nm}$ ) was about $23.9 \mathrm{~nm}$. This variation in height may be is an indication of undulations of the membrane surface resulting in nonuniform attachment and hence an apparent membrane thickness greater than the actual thickness of the membranes. These variations could have been caused at the loosely bound areas of the sample to the substrate due to disturbances caused by sonication.

To determine the dimension of the particles observed, we first plotted profiles of sections, which cut across several particles of similar sizes (Fig. 2c, e) on flatter regions of the membrane patch. The apparent diameter of the particles obtained from a height image was $21.4 \pm 3.3 \mathrm{~nm}$ (mean $\pm \mathrm{SD}, N=168$ ). The apparent diameter of those particles obtained from the phase image, defined as the distance between their steepest slope points in this case, was $22.0 \pm 2.4 \mathrm{~nm}$ (mean $\pm \mathrm{SD}, N=137$; Fig. 2e).

Because the apparent diameter depends on the contact geometry involving the object and the imaging tip of finite size ("tip-effect"), we assumed that both the particles and the tip were spherical. Then, the apparent diameter $\Delta$ of the particles can be given as

$\Delta=2 \sqrt{(R+r)^{2}-(R+h-r)^{2}}$

where $R$ is the radius of the tip and $r$ is the real radius of the particle embedded in the membrane up to a thickness $h$ (Fig. 3). This formula differs from a previously used correction [32, 39] because we assume that the $10-\mathrm{nm}$ particles are flush with the membrane at the external surface to be consistent with previous EM observations [29, 37]. The radius $R$ of oxide-sharpened fluid-scanning tips was estimated by the blind reconstruction method (see "Data acquisition and analysis" section and Fig. $2 \mathrm{~g}$ ). A sphere fit 

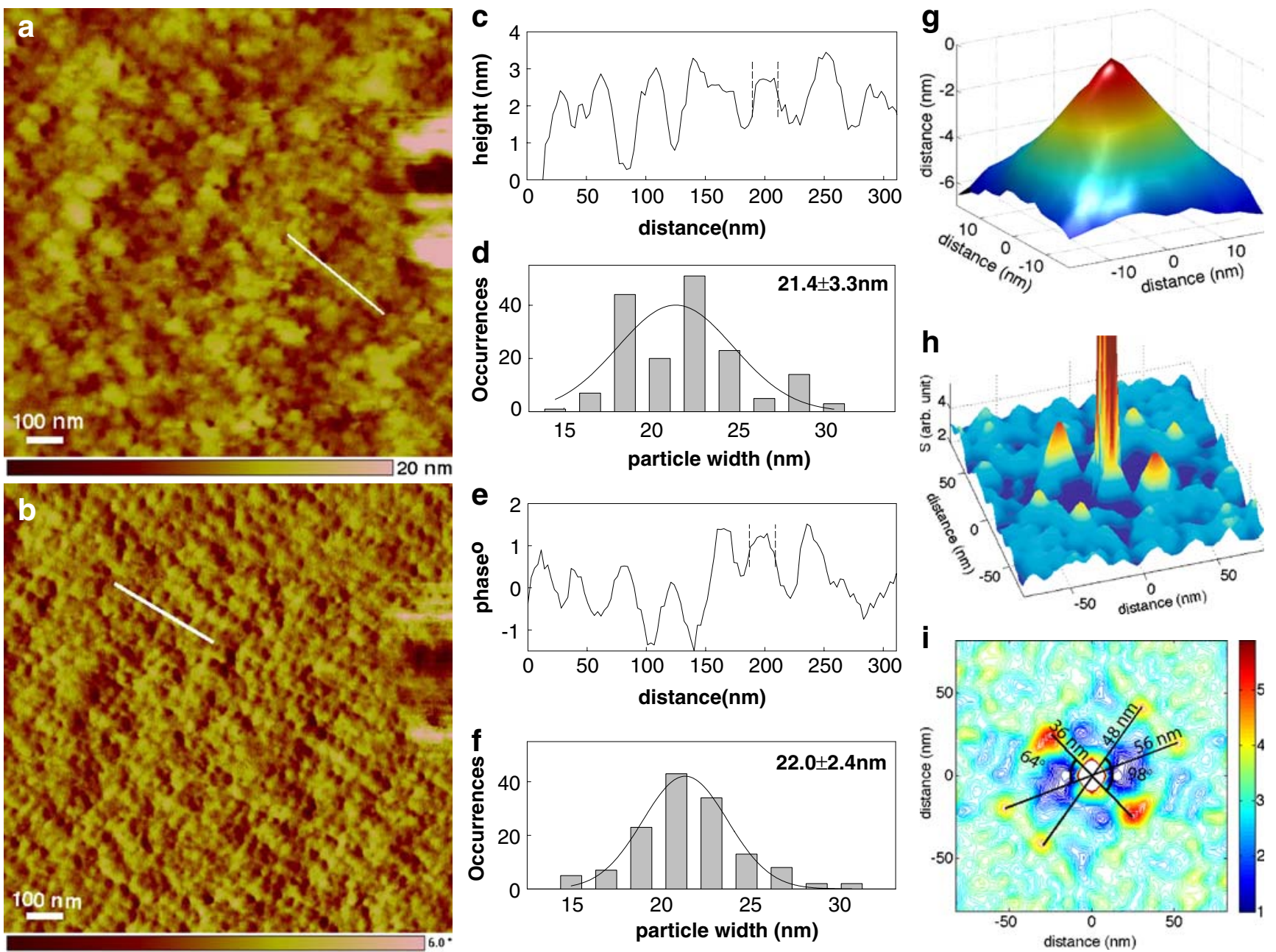

Fig. 2 The cytoplasmic face of the plasma membrane of an outer hair cell. a Topographic image and b phase image showing membraneassociated particles. The color coding of heights and phase contrast are shown under the respective images. c Height profile along the sectional line in image a. The distance between the two vertical dashed lines is $22 \mathrm{~nm}$. The average value is $21.4 \pm 3.3 \mathrm{~nm}$ obtained from different sections of the image a. d Particle size distribution obtained from sections of a. e Phase variation along the sectional line

(see "Materials and methods" section) to the estimated tips of several images gave an average value of $22.4 \pm 3.0 \mathrm{~nm}$ $(N=10)$. The estimated values utilized in our calculations are based on the three consecutive images that included Fig. 2a, where the average value was $19.0 \pm 2.0 \mathrm{~nm}$. Based on the minimum height of the free surface of the membrane, $h$ can be taken as $8.1 \mathrm{~nm}$ and the true diameter $2 r$ of the particles was estimated as $10.6 \pm 1.7 \mathrm{~nm}$, close to the values determined by electron microscopy $[29,58]$. The estimated diameter of the particles was not very sensitive to the tip radius. Our estimate would be $6 \%$ larger if $R=15 \mathrm{~nm}$ and $8 \%$ smaller if $R=30 \mathrm{~nm}$.

The reconstructed tip from the image (see "Materials and methods" section) had a large cone angle of $156^{\circ}$ presumably reflecting long-range nature of the interactions shown in b. The distance between the two vertical dashed lines is $22 \mathrm{~nm}$. The average value is $22.0 \pm 2.4 \mathrm{~nm}$ obtained from different sections of the image b. f Particle size distribution obtained from sections of $\mathbf{b}$. The solid lines in $\mathbf{d}$ and $\mathbf{e}$ are Gaussian fits to data. $\mathbf{g}$ Reconstructed tip shape. $\mathbf{h}$ The autocorrelation function limited to central regions for the reconstructed image of $\mathbf{b}$ (see "Data acquisition and analysis" section). i A contour plot of the autocorrelation function showing directions and angles of the main peaks 
the probe has with the sample in the aqueous medium. Particle density as determined by granulometric filters of ImageJ (see "Materials and methods" section) was $\sim 850 \mu^{-2}$. Given the large angle of the effective cone of the probe, it is likely that only those particles at the top are imaged, possibly underestimating the number of particles in the membrane. These imaged membrane particles appeared to be aligned (Fig. 2a, b). To study the distribution pattern, we calculated an autocorrelation function (see "Materials and methods" section), shown in Fig. 2h, i. There were two prominent peaks that are about $36 \mathrm{~nm}$ away from the center. The line connecting these two peaks agreed with the orientation of the alignment that we can detect by direct visual inspection of the image. The autocorrelation function confirmed linear alignment and indicated the main axis and mean spacing of $35.5 \pm 1.2 \mathrm{~nm}(N=16)$. This alignment is unlikely a scanning artifact because it is at approximately $45^{\circ}$ to the scanning axis. In addition to these peaks, there were less prominent peaks, a pair at an angle of $64^{\circ}$ from the main axis and at $48 \mathrm{~nm}$ from the center and another pair at a $98^{\circ}$ angle and a distance of $56 \mathrm{~nm}$ from the center. These three axes may be reminiscent of a hexagonal array. However, the angles that these axes formed deviated from $60^{\circ}$. In addition, peaks in the autocorrelation function were present only in the immediate proximity of the central peak. This differs from the autocorrelation function of a crystalline lattice, in which multiple peaks are regularly distributed. This difference indicates that the membrane particles that we observed had only short-range correlation and that they distributed rather randomly beyond their immediate neighbors.

\section{Antibody-labeled preparations}

Because prestin is the key protein for electromotility of the lateral membrane, we tested the immunoreactivity of our preparation using antibodies raised against polypeptides of the C-terminal region of prestin. To observe the geometry of the antibody complex formed as well as control on our preparation, we used actin antibody. The primary antibodies were conjugated to secondary antibodies to make them more readily recognizable (see "Materials and methods" section).

Six patches were identified from all the prestin-labeled preparations out of which four were selected for further analysis. One out of three patches was analyzed for the anti-actin sample while one out of three was analyzed for anti-DNASE II sample.

\section{Prestin antibody}

Previous immunofluorescence studies have shown that these antibodies label the cytosolic side [5]. Images of specimens labeled by prestin antibody (Fig. 4a) showed particles of $28.7 \pm 3.1 \mathrm{~nm}(N=100)$ in apparent diameter
(Fig. 4c, d), similar to the anti-actin-labeled specimens. For comparison with the unlabeled preparation, Fig. $4 \mathrm{~b}$ is presented in similar scale. These particles were taller than the $10-\mathrm{nm}$ particles in the unlabeled specimens by $2-3 \mathrm{~nm}$. The depth analysis performed over all sample for the unlabeled preparations gave a height of the particles as $1.3 \pm 0.3 \mathrm{~nm}$ $(N=5)$. Similar analysis for all samples for the antiprestinlabeled samples gave a value of $3.4 \pm 1.2 \mathrm{~nm}(N=15)$. This difference in height is statistically significant and is above the subnanometer resolution of AFM.

The membrane particles were aligned in one direction (Fig. 4a). The spacing between the particles of these prestin-labeled specimen was $38.7 \pm 2.7 \mathrm{~nm}(N=16)$, which was slightly larger than the spacing for the unlabeled preparation. However, unlike the unlabeled specimens, alignment in other directions was not clear by visual inspection. These images were not scanning artifacts because scanning the same area in different directions also produced similar results. This observation was confirmed by the autocorrelation function (Fig. 4f, g). The density of those particles was about $550 \mu^{-2}$, lower compared to the $850 \mu^{-2}$ for the $10-\mathrm{nm}$ particles in the unlabeled specimens. The low value for the antibody density could be due to steric hindrance between antibody complexes. The preservation of the main alignment pattern of 10-nm particles confirms specific binding of prestin antibodies to $10-\mathrm{nm}$ particles. With possible strain by antibody binding and lower density, the disappearance of weaker alignments may not be surprising. There are two alternative interpretations of the particles imaged in this preparation. One is that the $10-\mathrm{nm}$ particles are made larger by the binding of antibody complexes and another is that the particles imaged are primary and secondary antibody complexes. The issue of interpretations will be addressed in "Discussion" section.

The blind reconstruction method gave an overall average tip radius of $19.4 \pm 6.3 \mathrm{~nm}(N=10)$. The value obtained from three consecutive images shown in Fig. $4 \mathrm{e}$ is $12.5 \pm 2.5 \mathrm{~nm}$. If we can assume that the imaged particles are antibody complexes that are attached to the surface of the plasma membrane, then $h=0$ in Eq. 4. This interpretation leads to $8.2 \pm 2.4 \mathrm{~nm}$ as an estimate for the particle diameter using $12.5 \mathrm{~nm}$ for the radius of the tip.

\section{Actin antibody}

Anti-actin-labeled preparation was examined to determine whether the cytoskeleton, in which actin is the key component, is involved in the formation of the pattern observed in unlabeled specimen. The images obtained by AFM scanning (Fig. 5a, b) showed particles with an apparent diameter of $30.7 \pm 4.0 \mathrm{~nm}(N=181$; Fig. $5 \mathrm{c}$, d). The apparent height of the antibody complex is about $8 \mathrm{~nm}$ 

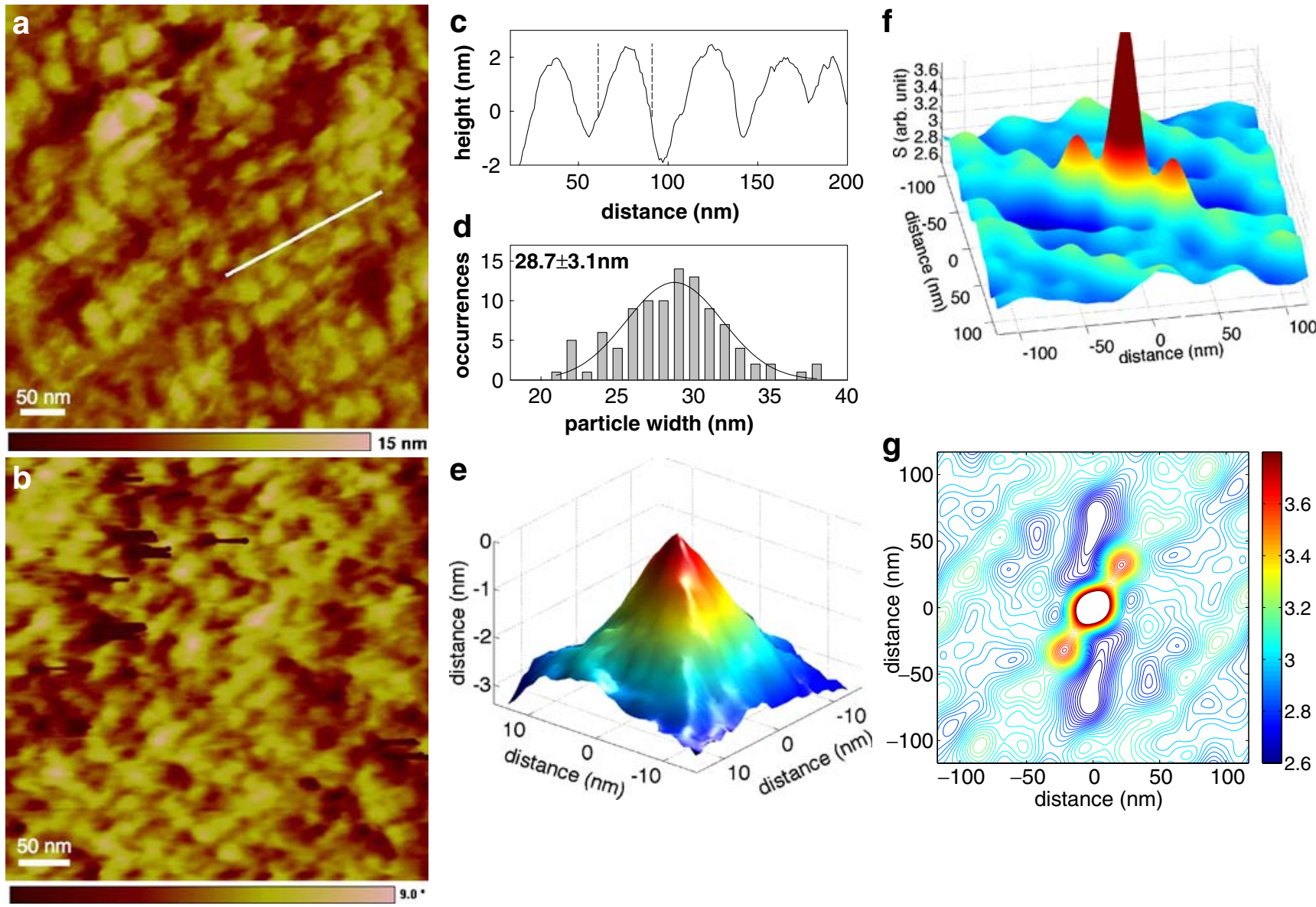

Fig. 4 a Topographic image of cytosolic side of OHC membrane treated with prestin antibodies followed by antirabbit secondary antibodies. b Phase image of unlabeled preparation for comparison (same scale). c Height profile along the sectional line in image a. The distance between the two vertical dashed lines is $28.7 \mathrm{~nm}$. The average value is $28.7 \pm 3.1 \mathrm{~nm}$ obtained from different sections of the image a. d

Particle size distribution. The solid line is a Gaussian fit to data. e Reconstructed tip shape. $\mathbf{f}$ The autocorrelation function limited to central regions for the background-subtracted gray scale image of a (see "Data acquisition and analysis" section). $\mathbf{g}$ The same autocorrelation function presented as contours

from the membrane surface, which is comparable to the thickness of the OHC plasma membrane. From depth analysis of the images, we obtained a value of $7.9 \pm$ $0.6 \mathrm{~nm}(N=19)$ for the height of these particles. The height of the antibody complex appears to indicate that the imaged particles are the antibodies that are attached to the top of the free surface of the plasma membrane including the $10-\mathrm{nm}$ particles. This height of the particles makes it unlikely that the particles imaged are the $10-\mathrm{nm}$ particles enlarged by the attachment of the antibodies. For this reason, the size correction formula should be $\Delta=2 \sqrt{(R+r)^{2}-(R-r)^{2}}$, which results from Eq. 4 when $h$ is set to zero. This equation gave a corrected value for the diameter of $11.8 \pm 4.7 \mathrm{~nm}$ based on a radius of $9.1 \pm 2.8 \mathrm{~nm}(N=7)$ for the probe tip (Fig. 5 e) obtained from seven consecutive images including Fig. 5a. The overall average radius was $13.5 \pm 4.8 \mathrm{~nm}$ obtained from 16 images (see "Materials and methods" section).

The number density was about $400 \mu \mathrm{m}^{-2}$, lower than the $850-\mu \mathrm{m}^{-2}$ density obtained for the unlabeled preparation and $550 \mu^{-2}$ obtained for the prestin-labeled preparation. The distribution of those particles appeared random (Fig. 5a, b) and markedly different from the unlabeled specimens. This observation was also confirmed by the autocorrelation function (Fig. $5 \mathrm{~g}$ ), where there is no clear peak other than the one in the center. Thus, the distribution of these particles did not reflect the alignment of the 10-nm particles. The randomness of the particle distribution cannot be due to the lower density. To prove this, we "reconstructed" (see "Materials and methods" section) an image on the basis of the particle locations of the antiprestin sample in which some of the particles were removed randomly to bring down the density comparable to the antiactin sample. In such a "reconstructed" image, the autocorrelation still showed the alignment pattern similar to that of antiprestin sample. 

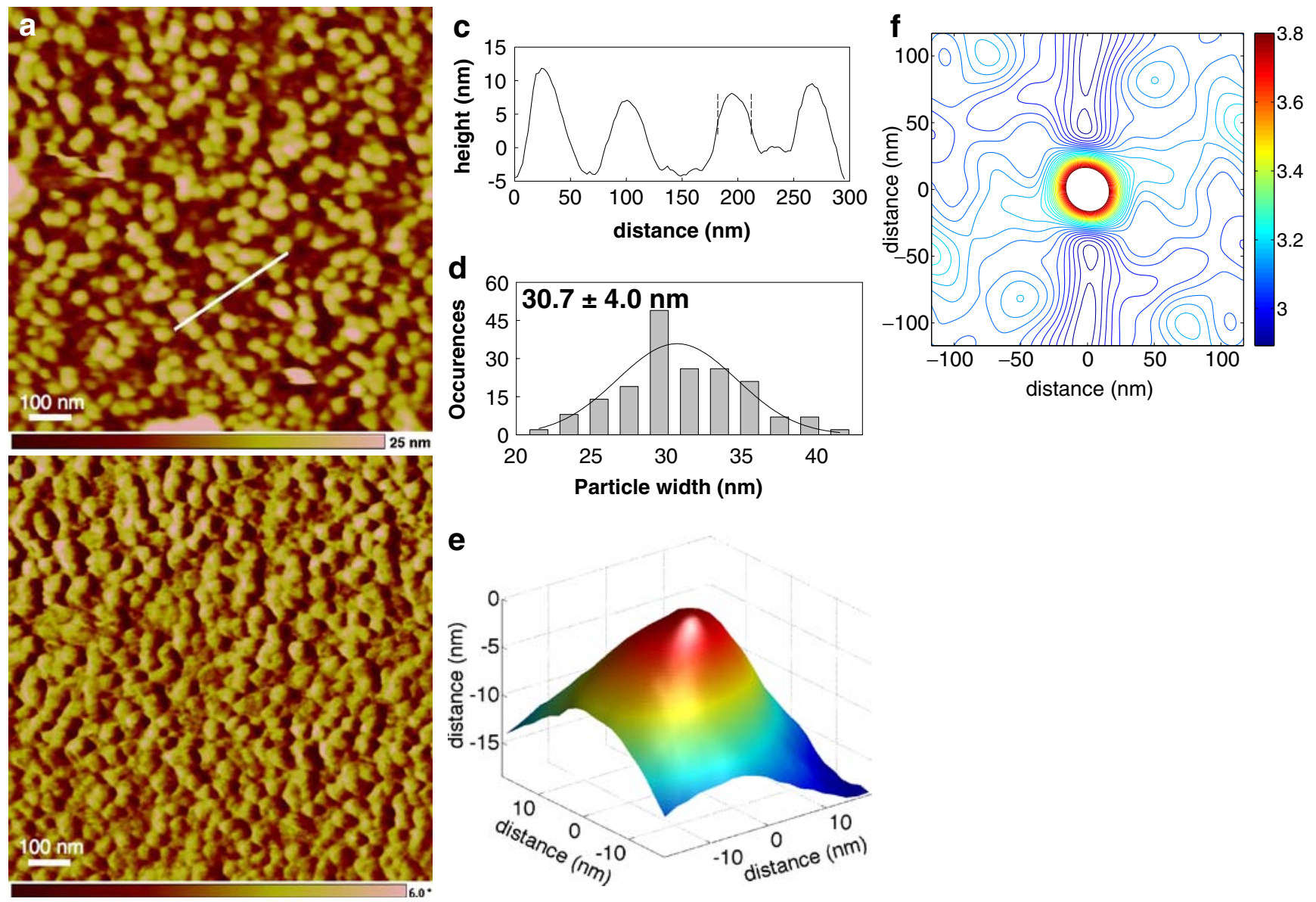

Fig. 5 Control: a topographic image of cytosolic side of $\mathrm{OHC}$ membrane patch treated with actin antibodies conjugated with secondary antibodies. b Corresponding phase image. c Height profile along the sectional line shown in image a. The distance between the two vertical dashed lines is $30.7 \mathrm{~nm}$. The average value is $30.7 \pm 4.0 \mathrm{~nm}$

obtained from different sections of the image b. d Particle size distribution. The solid line is a Gaussian fit to data. e Reconstructed tip shape. f A contour plot of the autocorrelation function of the background-subtracted gray scale image of a (see "Data acquisition and analysis" section)

\section{Control antibody}

\section{Discussion}

The distribution of particles in the anti-DNase II-treated specimens was also random and did not reproduce the alignment pattern of the 10-nm particles in the unlabeled preparation (not shown). Moreover, this distribution was similar to that in the anti-actin-labeled preparation. These observations confirm that the random distribution pattern of particles in anti-actin and anti-DNase II preparations indicates nonspecific interactions, and thus, these preparations serve as controls.

Unlike immunofluorescence, we found that nonspecific site blockers were not useful in our examination using AFM. BSA, for example, is unsuitable because it has the size comparable to membrane proteins. Instead, not using the nonspecific blockers had the advantage of actually observing the particles, presumably antibody complexes, and their positional organization at nanometer scale.

Antibody labeling

Our observations support the hypothesis that the 10-nm particles contain prestin because the antiprestin-labeled specimens retain features including alignment and spatial periodicity of the unlabeled specimens. These features are not shared by the anti-actin-labeled specimens. This contrast shows that the alignment that is exhibited by antibody-untreated preparation is based on prestin and is unrelated to the cytoskeleton. In addition, this result is consistent with the assumption that prestin is located in a regular manner in the 10-nm particles and not in the plasma membrane outside of the $10-\mathrm{nm}$ particles because the binding of antiprestin outside of the 10-nm particles could significantly disturb the alignment pattern of the unlabeled preparation. This point is highly relevant to the issue of prestin's oligomerization. Recent studies with gel electro- 
phoresis and FRET on prestin-transfected cells indicate oligomerization, most likely tetramerization of prestin [39, $40,58]$. However, oligomerization appears rather labile because removal and addition of cholesterol respectively reduces and increases oligomerization and the voltage sensitivity of prestin does not require oligomerization [42]. Certainly, tetramer formation appears consistent with the particle morphology indicated by CryoEM [37] and AFM [39]. How about the monomeric form? The existence of prestin monomers in the membrane of prestin-transfected cells can explain the FRET signal's sensitivity to cholesterol content [42]. Our observation does not suggest the presence of prestin outside of $10-\mathrm{nm}$ particles in our preparation. It is possible that antiprestin could not bind to monomeric prestin due to steric hindrance of 10-nm particles. If that possibility can be excluded, virtually all prestin molecules are in the $10-\mathrm{nm}$ particles in the plasma membrane of OHCs.

The unlabeled preparation shows a preferential axis with $35.5 \pm 1.2-\mathrm{nm}$ spacing. The antiprestin-labeled specimens show one alignment axis with $38.7 \pm 2.7-\mathrm{nm}$ spacing. Some stretching in the prominent axis and the disappearance of the less 11 prominent axis can be interpreted as result of disturbance due to antibody binding. Antibody binding may preserve regularity but may introduce some randomness, presumably eliminating weak alignment. The uneven structure may partly be causing minor differences in the alignment pattern. Roughness analysis showed that the maximum height range $\left(R_{\max }\right)$ is $20.0 \mathrm{~nm}$ for antiprestin-labeled preparation and $36.6 \mathrm{~nm}$ for anti-actin-labeled preparation.

Here, we consider two possible models of antibody binding. One is that (1) an antibody complex, including a primary antibody and secondary antibodies, spreads out relatively thin when it attaches to a surface and another is that (2) it takes a form that can be approximated by a sphere. The first model is based on the reports that the AFM image of a single antibody molecule attached to a substrate has an apparent diameter of $24 \mathrm{~nm}$ and a height of 2-3 $\mathrm{nm}$ [22] and that binding of antibody increases the height of an IP3 receptor by $2-3 \mathrm{~nm}$ [51]. The second model predicts a diameter of $9 \mathrm{~nm}$ based on the molecular weight of an antibody complex of $\sim 300 \mathrm{kDa}$ and the density of $\sim 1.35 \mathrm{~g} / \mathrm{cm}^{3}[20,31,49]$.

Our anti-actin-labeled preparation favored the second model. The elevation of the imaged particles is about $8 \mathrm{~nm}$, inconsistent with the first model and consistent with the second model. In addition, the estimated diameter of $11.8 \pm 4.7$ assuming the second model is consistent with the expected diameter. If we assume that actin antibodies bind to $10-\mathrm{nm}$ particles randomly in a manner consistent with the first model, the alignment pattern of the unlabeled preparation should be expected as it did in the antiprestin-labeled preparation. However, that is not the case.

Our antiprestin-labeled preparation, however, did not allow us to exclude either model. The elevation of $3 \mathrm{~nm}$ above the plasma membrane is consistent with the first model. Although this elevation may not be consistent with the second model, the estimated diameter of $8.2 \pm 2.4 \mathrm{~nm}$ for the antiprestin complex based on the second model is still consistent with this interpretation. It could be speculated based on the second model that prestin antibodies might be bound to a particular site on the 10-nm particle presumably close to the membrane surface and that actin antibodies, which bind to the plasma membrane randomly, are more elevated.

It is likely that the AFM image of antibody complexes may depend on the surface to which they bind. Our observation on anti-actin-labeled preparation excludes the first model whereas previous reports [22, 51], which appear to have strong effects of glass and mica surface on antibody binding, exclude the second model.

Ten-nanometer particles and their alignment in the membrane

The particles in our unlabeled preparation have a horizontal diameter of $10 \mathrm{~nm}$ after correction. This value is consistent with the 10-nm particles reported by EM studies [29, 45]. The height of these particles is about $12 \mathrm{~nm}$ from the substrate, which is consistent with a recent EM study [37]. This neural network analysis of EM images also show that these particles must protrude into the cytosolic side of the membrane. Such structure is consistent with the substantial cytosolic moiety, particularly near the $\mathrm{C}$ terminus of prestin, predicted by several reports [10, 40, 57]. This is also consistent with a freeze-fracture image showing dense particles protruding on the cytosolic face of the plasma membrane (B. Kachar, personal communication).

However, the value of $850 \mu^{-2}$ for the density of the $10-\mathrm{nm}$ particles in our preparation is lower than those obtained from EM studies, which range from 2,500 [29] to $6,000 \mu^{-2}$ [17]. It is likely that $6,000 \mu \mathrm{m}^{-2}$ is an overestimate because it includes particles of two different sizes [17]. Those values are somewhat lower than the density, typically between 7,000 and $10,000 \mu^{-2}$, of functional motor unit determined from nonlinear capacitance, assuming a two-state model [29, 45]. A lower bound of density estimates based on nonlinear capacitance data would be $\sim 2,000 \mu \mathrm{m}^{-2}$ assuming that the functional unit is prestin monomer and the $10-\mathrm{nm}$ particles are tetramers of prestin. Our lower value could be due to undulation of the sample surface. The value for the maximum height range $\left(R_{\max }\right), 23.9 \mathrm{~nm}$, obtained from roughness analysis is two times greater than the height of the membrane particles, in the unlabeled preparation. Undulation of the sample may have prevented the steeply tapered tip geometry (Fig. 4e) from reaching (and thus imaging) all membrane particles. However, this effect does not appear to be significant for the unlabeled preparation because a hexagonal packing 
with 35.5-nm spacing leads to the density of $920 \mu \mathrm{m}^{-2}$, not significantly larger than the observed density of $850 \mu^{-2}$. Thus, the lower density of $10-\mathrm{nm}$ particle is more likely due to their dislodging during sonication or the effect of the medium we used, which is high in $\mathrm{Cl}^{-}$and $\mathrm{Ca}^{2+}$ concentrations.

The effect of the steeply tapered tip appears to have more significant effect on imaging the antibody-labeled preparation where the particle density of $550 \mu^{-2}$ has a larger difference from the density of $770 \mu^{-2}$ obtained by assuming a hexagonal packing with $38.7-\mathrm{nm}$ spacing.

The spacing of $36 \mathrm{~nm}$ between the $10-\mathrm{nm}$ particles in the unlabeled preparation is reminiscent of the half-period of the actin double helix [26]. However, the structure observed cannot be due to the presence of outer hair cell cytoskeleton because labeling with actin antibody does not show such regularity. In addition, we do not see any resemblance to the structure observed in the AFM studies of outer hair cell cytoskeleton either by AFM [54] or by electron microscopy [25]. These studies show that the OHC cytoskeleton comprises of parallel actin filaments 30 to $80 \mathrm{~nm}$ apart cross-linked by spectrin. The actin-spectrin network is connected to the plasma membrane via 25 -nm-long pillars of unknown composition defining a height of at least $25 \mathrm{~nm}$ for the patch from a substrate. A recent publication also showed that cytoskeleton elements do not directly interact with prestin [34]. The heights of our membrane patches are different from these values suggesting that our membrane patches do not contain structure in our preparation.

In our preparation, the 10-nm particles showed a distinct alignment pattern. In comparison, EM studies are not unanimous regarding the alignment of the 10-nm particles [17, 29, 45]. Random distribution is observed in glutaraldehydetreated preparations [45]. However, glutaraldehyde does not necessarily induce randomness because hexagonal patterns are observed in freeze fracture preparations with [17] or without [29] glutaraldehyde fixation. We compare our results with the freeze fracture EM images (without fixation) [29].

The EM images show semicrystalline regularity and equally strong alignment in two directions, which form a $68^{\circ}$ angle. Although our images have symmetry axes similar to those preparations, alignment is more significant along one axis, and the correlation length (the extent of the ordered regions) is much shorter. Large membrane undulation and sonication uprooting might partly be responsible for the short correlation length. One possible factor responsible for the difference in the alignment pattern could be glutaraldehyde treatment. Another factor could be the $\mathrm{Cl}^{-}-$and $\mathrm{Ca}^{2+}$-rich medium that we used. However, perhaps the most likely factor for the difference in the alignment pattern would be the difference in the density of the 10-nm particles.

What is the origin of the alignment observed? It has been shown that anisotropy of the lateral wall in OHCs is based on the cortical cytoskeleton $[17,24,25]$. It would be possible that the cytoskeleton and membrane proteins associated with it could orient membrane proteins if the interactions between them are solid like. As we have discussed, our result obtained with actin antibodies revealed that the alignment pattern is not based on actin, the primary component of the cytoskeleton. We did not detect in our preparation a regular pattern that matches the description of "pillars" that are thought to connect the plasma membrane with actin filaments $[17,25]$. In addition, solid-like interaction involving prestin is very

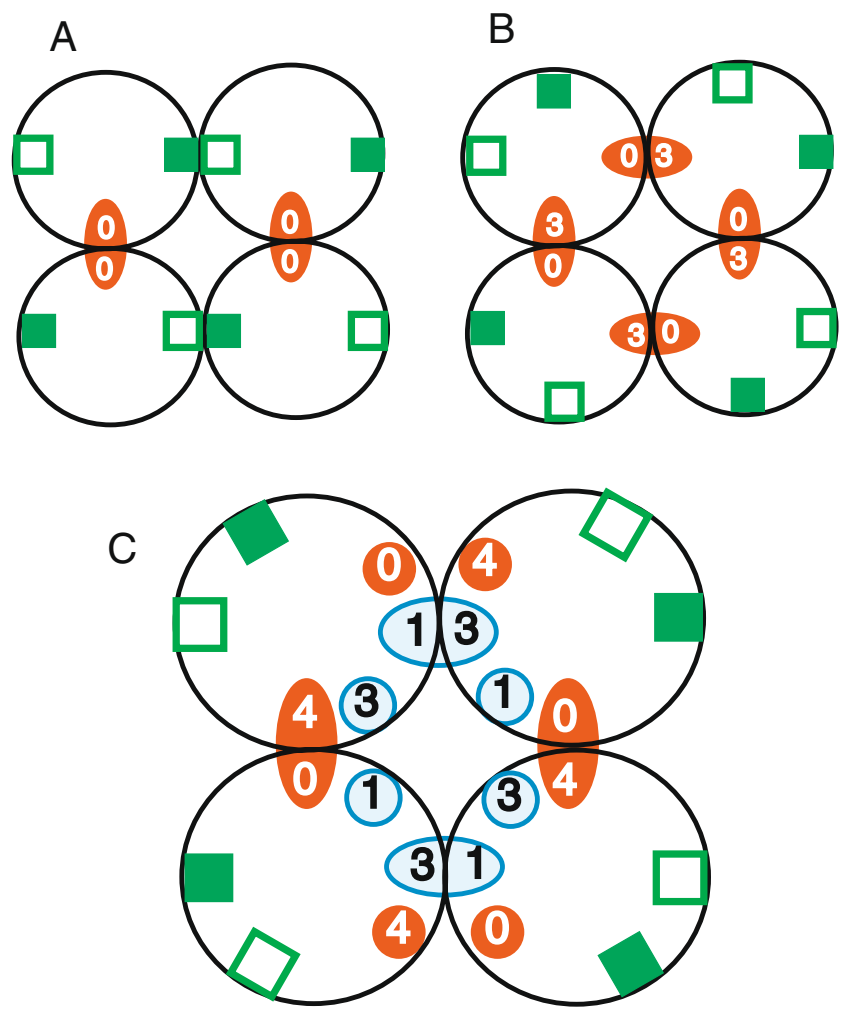

Fig. 6 Models for the 10-nm particle internal organization. Here, prestin molecules in the membrane are schematically represented by circles. Consider two kinds of bonds. One is strong, such as disulfide bond, which is involved in dimerization (shown in red), and the other is weak and responsible for alignment (green). a A model in which strong bonds are formed between the identical sites. This model leads to a linear polymer rather than tetramers because bonds (open and filled green squares) formed between two dimers should also form with the next dimers on the right and left. b A tetramer model in which strong bonds are formed between nonidentical sites 0 and 3, which are $90^{\circ}$ apart. The residues exposed to the right and left of the tetramer, symbolically represented by open and closed green squares, are the same as those in the up-down direction. This results in a square lattice, contradicting preferential alignment in one direction. c A tetramer model with strong dimerizing bonds between sites 0 and 4 , which are $120^{\circ}$ apart. Yet another types of strong bonds are between site 1 and 3. The residues exposed to the right and left of the tetramer, symbolically represented by open and closed green squares, are different from those in the up-down direction. This tetramer can have a preferential direction of alignment, consistent with our observation, and also a symmetry compatible with hexagonal packing 
unlikely because prestin diffuses easily along the plasma membrane [44]. The diffusion coefficient of prestin is in the range $0.08-0.35 \mu \mathrm{m}^{2} \mathrm{~s}^{-1}$ [44], much higher than the value $0.0001 \mu^{2} \mathrm{~s}^{-1}$ for $\mathrm{Na}^{+}$channels in skeletal muscle [16]. For those reasons, the observed alignment may reflect symmetries in the internal organization of these particles. For example, hexagonal packing of purple membrane seems to reflect trimer formation of bacteriorhodopsin $[18,46,50]$. The square lattice of aquaporin corresponds to the molecule's tetramerization [55].

We made an attempt at explaining the particle alignment that we observed, assuming that each $10-\mathrm{nm}$ particle is a homologous tetramer of prestin molecules to illustrate a possible internal symmetry of these particles. The reason for this assumption is that it is the simplest. It has been pointed out that homologous oligomerization may lead to a reduction in voltage sensitivity of electromotility due to increased charge interaction [36]. Heterologous oligomers may not suffer such an effect. Indeed, we do not have any reason to reject the possibility of heterologous oligomers. Currently, however, we have no data to construct any heterologous model. A homologous tetramer is consistent with previous reports $[11,36,37,39,40,42,47,58]$ and with the dimension of a $10-\mathrm{nm}$ particle, which should have about $300-\mathrm{kDa}$ mass assuming a typical value for protein density is closest to a tetramer $(4 \times 81 \mathrm{kDa})$. Previous studies show that some disulfide bonds are important for the functionality of prestin [52], although they are not required for dimerization as observed by gel electrophoresis [11].

We can reject some models for the 10-nm particles being inconsistent with forming the observed alignment (Fig. 6). We can also show a possible model that satisfies linear alignment and includes prestin dimerization by a disulfide bond [52, 58]. It has two types of interaction to form dimers (Fig. 6). This model is intended as an illustration of possible significance of internal symmetry in determining particle alignment. It is quite possible that the structure of $10 \mathrm{~nm}$ could be more complex involving proteins other than prestin.

Finally, the cell-free preparation formed from $\mathrm{OHCs}$ that we described would be useful in further studying the $10-\mathrm{nm}$ particles and oligomerization of prestin. The uniform density and alignment of the $10-\mathrm{nm}$ particles appear to make the cell-free preparation of OHCs logical for examining the effect of factors that control oligomerization, particularly that of cholesterol. We plan to address this issue by improving our preparation method.

\section{Conclusions}

Our study shows that AFM is a useful method in studying the structure of the plasma membrane when combined with cellfree preparations. We showed that the cytosolic face of the plasma membrane of the lateral wall of $\mathrm{OHCs}$ has particles that match the 10-nm particles reported by EM studies. We further provided evidence that these particles have immunoreactivity to prestin antibodies. These observations indicate that the 10-nm particles are a focus of further studies to clarify structure-function relationship of electromotility. The combination of a cell-free preparation and AFM could be useful methods for such studies. We may need to improve the cellfree preparation to minimize disturbance to membrane proteins to address the issue regarding packing density and pattern of these particles as well as to make the method viable for functional studies.

Acknowledgments We thank Dr. Bechara Kachar for kindly providing us with prestin antibodies. We also thank Drs. Ronald Petralia and Paul Gallant for useful comments. This work is supported by the intramural research program of NIDCD, NIH.

Open Access This article is distributed under the terms of the Creative Commons Attribution Noncommercial License which permits any noncommercial use, distribution, and reproduction in any medium, provided the original author(s) and source are credited.

\section{References}

1. Adachi M, Iwasa KH (1999) Electrically driven motor in the outer hair cell: effect of a mechanical constraint. Proc Natl Acad Sci USA 96:7244-7249

2. Ashmore JF (1987) A fast motile response in guinea-pig outer hair cells: the molecular basis of the cochlear amplifier. J Physiol (Lond) 388:323-347

3. Ashmore JF (1990) Forward and reverse transduction in guineapig outer hair cells: the cellular basis of the cochlear amplifier. Neurosci Res Suppl 12:S39-S50

4. Avery J, Ellis DJ, Lang T, Holroyd P, Riedel D, Henderson RM, Edwardson JM, Jahn R (2000) A cell-free system for regulated exocytosis in pc12 cells. J Cell Biol 148:317-324

5. Belyantseva IA, Adler HJ, Curi R, Frolenkov GI, Kachar B (2000) Expression and localization of prestin and the sugar transporter glut-5 during development of electromotility in cochlear outer hair cells. J Neurosci 20:RC116

6. Berg JM, Tymoczko JL, Stryer L, Clarke ND (2002) Biochemistry, 5 th edn. Freeman, New York

7. Brownell W, Bader C, Bertrand D, Ribaupierre Y (1985) Evoked mechanical responses of isolated outer hair cells. Science 227:194-196

8. Chan DK, Hudspeth AJ (2005) $\mathrm{Ca}^{2+}$ current-driven nonlinear amplification by the mammalian cochlea in vitro. Nature Neurosci 8:149-155

9. Dallos P, Wu X, Cheatham MA, Gao J, Zheng J, Anderson CT, Jia S, Wang X, Cheng WHY, Sengupta S, He DZZ, Zuo J (2008) Prestin-based outer hair cell motility is necessary for mammalian cochlear amplification. Neuron 58(3):333-339

10. Déak L, Zheng J, Orem A, Du GG, Aguinaga S, Matsuda K, Dallos P (2005) Effects of cyclic nucleotides on the function of prestin. J Physiol (Lond) 563:483-496

11. Detro-Dassen S, Schnzler M, Lauks H, Martin I, zu Berstenhorst SM, Nothmann D, Torres-Salazar D, Hidalgo P, Schmalzing G, Fahlke C (2008) Conserved dimeric subunit stoichiometry of slc26 multifunctional anion exchangers. J Biol Chem 283(7):4177-4188 
12. Dong XX, Iwasa KH (2004) Tension sensitivity of prestin: comparison with the membrane motor in outer hair cells. Biophys J 86:1201-1208

13. Dong XX, Ehrenstein D, Iwasa KH (2000) Fluctuation of motor charge in the lateral membrane of the cochlear outer hair cell. Biophys J 79:1876-1882

14. Dong XX, Ospeck M, Iwasa KH (2002) Piezoelectric reciprocal relationship of the membrane motor in the cochlear outer hair cell. Biophys J 82:1254-1259

15. Dongmo S, Troyon M, Vautrot P, Delain E, Bonnet N (1996) Blind restoration method of scanning tunneling and atomic force microscopy images. J Vac Sci Technol B 14(2):1552-1556

16. Edidin M (1987) Rotational and lateral diffusion of membrane proteins and lipids: phenomena and function. Curr Top Membr Transp 29:91-127

17. Forge A (1991) Structural features of the lateral walls in mammalian cochlear outer hair cells. Cell Tissue Res 265(3):473-483

18. Fotiadis D, Engel A (2004) High-resolution imaging of bacteriorhodopsin by atomic force microscopy. Methods Mol Biol 242:291-303

19. Gale JE, Ashmore JF (1994) Charge displacement induced by rapid stretch in the basolateral membrane of the guinea-pig outer hair cell. Proc Roy Soc (Lond) B Biol Sci 255:233-249

20. Gekko K, Noguchi H (1979) Compressibility of globular proteins in water at $25^{\circ} \mathrm{C}$. J Phys Chem 83:2706-2714

21. Gulley RL, Reese TS (1977) Regional specialization of the hair cell plasmalemma in the organ of Corti. Anat Rec 189:109-124

22. Hansma HG (1999) Varieties of imaging with scanning probe microscopes. Proc Natl Acad Sci USA 96(26):14,678-14,680

23. Heuser J (2000) The production of 'cell cortices' for light and electron microscopy. Traffic 1(7):545-552

24. Holley MC (1996) Outer hair cell motility. In: Dallos P, Popper AN, Fay RR (eds) The cochlea. Springer, New York, pp 386-434

25. Holley MC, Kalinec F, Kachar B (1992) Structure of the cortical cytoskeleton in mammalian outer hair cells. J Cell Sci 102(3):569-580

26. Howard J (2001) Mechanics of motor proteins and the cytoskeleton. Sinauer Associates, Sunderland

27. Iwasa KH (1993) Effect of stress on the membrane capacitance of the auditory outer hair cell. Biophys J 65:492-498

28. Kachar B, Brownell WE, Altschuler R, Fex J (1986) Electrokinetic shape changes of cochlear outer hair cells. Nature 322:365-368

29. Kalinec F, Holley M, Iwasa KH, Lim DJ, Kachar B (1992) A membrane-based force generation mechanism in auditory sensory cells. Proc Natl Acad Sci USA 89:8671-8675

30. Kennedy HJ, Crawford AC, Fettiplace R (2005) Force generation by mammalian hair bundles supports a role in cochlear amplification. Nature 433(7028):880-883

31. Kuntz ID, Kauzmann W (1974) Hydration of proteins and polypeptides. Adv Protein Chem 28:239-345

32. Larmer J, Schneider SW, Danker T, Schwab A, Oberleithner H (1997) Imaging excised apical plasma membrane patches of MDCK cells in physiological conditions with atomic force microscopy. Pflügers Arch 434(3):254-260

33. Le Grimellec C, Giocondi MC, Lenoir M, Vater M, Sposito G, Pujol R (2002) High resolution three-dimensional imaging of the lateral plasma membrane of cochlear outer hair cells by atomic force microscopy. J Comp Neurol 451:62-69

34. Legendre K, Safieddine S, Kssel-Andermann P, Petit C, ElAmraoui A (2008) II-V spectrin bridges the plasma membrane and cortical lattice in the lateral wall of the auditory outer hair cells. J Cell Sci 121(20):3347-3356

35. Liberman MC, Gao J, He DZ, Wu X, Jia S, Zuo J (2002) Prestin is required for electromotility of the outer hair cell and for the cochlear amplifier. Nature 419:300-304

36. Mcguire R, Pereira F, Raphael R (2008) Effects of cysteine mutations on prestin function and oligomerization. Assoc Res Otolaryngol. 31st Midwinter Meeting Abstracts
37. Mio K, Kubo Y, Ogura T, Yamamoto T, Arisaka F, Sato C (2008) The motor protein prestin is a bullet-shaped molecule with inner cavities. J Biol Chem 283(2):1137-1145

38. Mountain DC, Hubbard AE (1994) A piezoelectric model of outer hair cell function. J Acoust Soc Am 95:350-354

39. Murakoshi M, Gomi T, Iida K, Kumano S, Tsumoto K, Kumagai I, Ikeda K, Kobayashi T, Wada H (2006) Imaging by atomic force microscopy of the plasma membrane of prestin-transfected Chinese hamster ovary cells. J Assoc Res Otolaryngol 7(3):267-278

40. Navaratnam D, Bai JP, Samaranayake H, Santos-Sacchi J (2005) $\mathrm{N}$-terminal-mediated homomultimerization of prestin, the outer hair cell motor protein. Biophys J 89(5):3345-3352

41. Oliver D, He DZ, Klocker N, Ludwig J, Schulte U, Waldegger S, Ruppersberg JP, Dallos P, Fakler B (2001) Intracellular anions as the voltage sensor of prestin, the outer hair cell motor protein. Science 292 (5525):2340-2343

42. Rajagopalan L, Greeson JN, Xia A, Liu H, Sturm A, Raphael RM, Davidson AL, Oghalai JS, Pereira FA, Brownell WE (2007) Tuning of the outer hair cell motor by membrane cholesterol. J Biol Chem 282(50):36,659-36,670

43. Santos-Sacchi J (1991) Reversible inhibition of voltage-dependent outer hair cell motility and capacitance. J Neurosci 11:3096-3110

44. Santos-Sacchi J, Zhao HB (2003) Excitation of fluorescent dyes inactivates the outer hair cell integral membrane motor protein prestin and betrays its lateral mobility. Pflugers Arch 446(5):617-622

45. Santos-Sacchi J, Kakehata S, Kikuchi T, Katori Y, Takasaka T (1998) Density of motility-related charge in the outer hair cell of the guinea pig is inversely related to best frequency. Neurosci Lett 256:155-158

46. Scheuring S, Müller DJ, Stahlberg H, Engel HA, Engel A (2002) Sampling the conformational space of membrane protein surfaces with the AFM. Eur Biophys J 31(3):172-178

47. Sfondouris J, Rajagopalan L, Pereira FA, Brownell WE (2008) Membrane composition modulates prestin-associated charge movement. J Biol Chem 283(33):22,473-22,481

48. Sonka M, Hlavac V, Boyle R (1999) Image processing, analysis, and machine vision, 2nd edn. PWS, New York

49. Squire PG, Himmel ME (1979) Hydrodynamics and protein hydration. Arch Biochem Biophys 196(1):165-177

50. Stark M, Möller C, Muller DJ, Guckenberger R (2001) From images to interactions: high-resolution phase imaging in tappingmode atomic force microscopy. Biophys J 80(6):3009-3018

51. Suhara W, Kobayashi M, Sagara H, Hamada K, Goto T, Fujimoto I, Torimitsu K, Mikoshiba K (2006) Visualization of inositol 1, 4, 5 -trisphosphate receptor by atomic force microscopy. Neurosci Lett 391(3):102-107

52. Surguchev A, Bai JP, Bian S, Santos-Sacchi J, Navaratnam D (2009) Not all cysteines are created equal in prestin. Assoc Res Otolaryngol. 32nd Midwinter Meeting Abstracts

53. Villarrubia JS (1994) Morphological estimation of tip geometry for scanned probe microscopy. Surf Sci 321(3):287-300

54. Wada H, Kimura K, Gomi T, Sugawara M, Katori Y, Kakehata S, Ikeda K, Kobayashi T (2004) Imaging of the cortical cytoskeleton of guinea pig outer hair cells using atomic force microscopy. Hear Res 187(1-2):51-62

55. Walz T, Smith BL, Agre P, Engel A (1994) The three-dimensional structure of human erythrocyte aquaporin chip. EMBO J 13 (13):2985-2993

56. Williams PM, Shakesheff KM, Davies MC, Jackson DE, Roberts CJ, Tendler SJB (1996) Blind reconstruction of scanning probe image data. J Vac Sci Technol B 14(2):1557-1562

57. Zheng J, Shen W, He DZZ, Long KB, Madison LD, Dallos P (2000) Prestin is the motor protein of cochlear outer hair cells. Nature 405:149-155

58. Zheng J, Du GG, Anderson CT, Keller JP, Orem A, Dallos P, Cheatham M (2006) Analysis of the oligomeric structure of the motor protein prestin. J Biol Chem 281(29):19,916-19,924 